\title{
Facebook. Una herramienta 2.0 al servicio de la docencia universitaria
}

\section{Facebook. A 2.0 tool at the service of university teaching.}

\author{
PIEDAD VARGAS-SORIA \\ Departamento de Didáctica de la Expresión \\ Musical, Plástica y Corporal. Universidad de Valladolid. \\ (D) https://orcid.org/0000-0002-0383-4313
}

BLANCA GARCÍA-GÓMEZ

Departamento de Organización de empresas y comercialización e investigación de mercados. Universidad de Valladolid.

\section{ELENA JIMÉNEZ-GARCÍA}

Departamento de Didáctica de la Lengua y la Literatura. Universidad de Valladolid.

(D) https://orcid.org/0000-0002-2410-9170

\section{RESUMEN}

El sistema de enseñanza ha sufrido un cambio sustancial, se ha pasado de una enseñanza 1.0, centrada en el profesor como transmisor de conocimiento, a un sistema 2.0 en el que el estudiante asume un rol activo. Incluso se puede decir que estamos en un escenario 3.0, donde la comunicación se manifiesta en todas direcciones, con un creciente protagonismo del intercambio de conocimiento entre iguales a través de una enseñanza participativa y colaborativa. La implementación de este sistema exige nuevos medios y es ahí donde el empleo de las redes sociales cobra protagonismo en el proceso de enseñanza-aprendizaje. Facebook se revela como la más popular entre los estudiantes universitarios. Su aplicación en docencia a través de esta experiencia de innovación, ha permitido medir el positivo impacto de su utilización en la adquisición de competencias en el Grado en Administración y Dirección de Empresas, pero extensibles a otros estudios universitarios.

\section{ABSTRACT}

The education system has undergone a substantial change, it has gone from a teaching 1.0, centered on the teacher as a transmitter of knowledge, to a 2.0 system in which the student assumes an active role. Moreover we could even say that we are in a scenario 3.0, where communication manifests itself in all directions, with a growing role of the exchange of knowledge among equals through participatory and collaborative teaching. The implementation of this system requires new means and this is where the use of social networks takes center of the stage in the teachinglearning process. Facebook is revealed as the most popular social media among university students. Its application in teaching through this experience of innovation, has allowed to measure the positive impact of its use in the acquisition of competences in the Degree in Business Administration and Management. This could be applied extendable to other university studies.

Para citar este artículo: Vargas-Soria, P., García-Gómez, B. y Jiménez-García, E. (2019). Facebook. Una herramienta 2.0 al servicio de la docencia universitaria. EA, Escuela Abierta, 22, 15-27. doi:10.29257/EA22.2019.03

KEYWORDS

Facebook, Teaching, University, Social Networks, Collaborative Learning, .
Recibido: 09/11/18

Aceptado: 04/06/19

PALABRAS CLAVES

Facebook, docencia, universidad, redes sociales, aprendizaje colaborativo. 


\section{INTRODUCCIÓN Y ESTADO DE LA CUESTIÓN}

\subsection{Redes sociales y la nueva enseñanza universitaria}

El cambio en la forma de concebir la enseñanza universitaria derivado del Espacio Europeo de Educación Superior ha supuesto, entre otras modificaciones, un notable incremento en la dedicación no presencial de los estudiantes. Precisamente esta necesidad de incrementar las horas de trabajo fuera del aula exige, tanto la implementación de nuevas metodologías, como el uso de herramientas que faciliten la cooperación y la comunicación dentro del proceso de aprendizaje (Saquete, Garrigós, Mazón e Izquierdo, 2011). Ortego, Jiménez y Fernández (2015) consideran que ha surgido un nuevo enfoque de comunicación e información y que las redes sociales han ganado popularidad, especialmente entre los adolescentes y adultos jóvenes, quienes muestran una actitud tan favorable que los han incluido en su rutina diaria. De hecho, las redes sociales son el canal ideal para compartir datos y conocimiento de una manera rápida, cómoda y fácil, y como docentes, debemos aprovecharlas para proporcionar herramientas y nuevas formas de interacción para abordar nuevos desafíos. Por ejemplo, algunos de sus puntos potenciales, como la colaboración, el libre flujo de información y la generación de contenido para la construcción de conocimiento se han aplicado recientemente a la educación (De Haro, 2010). Sin embargo Selwyn (2009) y Mazer y otros (citados en Balula y Moreira, 2014), defienden que algunas de las cualidades de las redes sociales, en algunos contextos educativos, pueden entrar en conflicto con paradigmas pedagógicos tradicionales, ya que estas redes son canales informales de comunicación y espacios poco estructurados para actividades académicas, y los docentes pueden quebrantar las expectativas de los estudiantes al usar Facebook. Por el contrario, Junco (2011) y Amador y Amador (2014) consideran que el uso de esta red social en contextos académicos resulta positivo en el rendimiento académico de los estudiantes.

Por otro lado, el aprendizaje basado en competencias exige al docente y al estudiante un esfuerzo tendente a comprender cuál es el rol que cada uno ha de desarrollar dentro del proceso didáctico. Como un ingrediente más, la escasez de medios materiales unida a la soledad de un profesorado que, sin orientación alguna, ha tenido que adaptar sus herramientas a un escenario nuevo, han constituido la punta de lanza en la investigación de medios al servicio de nuevos planteamientos docentes. En definitiva, asistimos a un cambio de paradigma educativo, unido a un cambio metodológico que ha de potenciar el papel activo del estudiante, su iniciativa y el pensamiento crítico (Esteve, 2009).

En medio de este proceso de cambio emergen las redes sociales como una herramienta de incuestionable notoriedad entre los estudiantes universitarios. No en vano, la popularidad que están alcanzando ha hecho que trasciendan el objetivo lúdico con el que nacieron, pasando a convertirse en herramientas de uso habitual en numerosas actividades laborales y educativas (Tomás y Navarro, 2011). Precisamente, en esta línea, muchos son los estudios que proponen la utilidad de las redes sociales como herramientas de trabajo colaborativo, capaces de mejorar el rendimiento académico de las horas no presenciales (León de Mora, 2010 y Saquete y otros, 2011). Además de la familiaridad del estudiante con estos instrumentos, su ilimitada disponibilidad en el tiempo y en el espacio, junto a su carácter interactivo, son sólo algunos de los factores determinantes de su cada vez mayor empleo en el aula. Y es que, en medio del cambio de paradigma al que nos referíamos en líneas precedentes, las redes sociales pueden jugar un importante papel en la tarea de potenciar la motivación y participación del alumnado, tanto en el proceso de aprendizaje, como en la colaboración e intercambio de información (Garrigós, Mazón, Saquete, Puchol y Moreda, 2010).

De acuerdo con Ponce (2012), hablamos de redes sociales para referirnos a aquellas estructuras compuestas por un grupo de personas que comparten un interés común, relación o actividad a través de Internet, siendo precisa- 
mente en la web donde tienen lugar los encuentros sociales, en tiempo real o diferido. Es decir, consideramos que una red social es una herramienta diseñada para crear espacios capaces de promover o facilitar la formación de comunidades e instancias de intercambio social (Cobo y Pardo, 2007).

En general, las redes sociales brindan a los usuarios una forma de interacción social abierta (Tomás y Navarro, 2011), en la que pasan de ser meros receptores de información a ser productores y a través de la que aprenden unos de otros, compartiendo una compilación de recursos (Lozano, 2004). Precisamente esta es otra de las utilidades que aportan de cara a la formación de los estudiantes en competencias. Pues bien, hoy la educación universitaria cambia el rol del estudiante del mismo modo que lo hacen las redes sociales (Valenzuela, 2013): los nuevos modelos de aprendizaje pasan por el incremento del protagonismo e iniciativa del estudiante, en detrimento de la clase magistral. Además, estamos siendo testigos de un cambio de dirección en la práctica docente, de un modelo bidireccional (tutoría) o, en ocasiones unidireccional (lección magistral) pasamos a otro de carácter colaborativo, en el que emergen elementos como la evaluación entre iguales o el trabajo en equipo. Precisamente en esta línea (Dapía y Escudero, 2014) reconocen la capacidad de las redes sociales para superar paradigmas centrados en la lección magistral que promueven la participación, la interacción y la colaboración como bases del aprendizaje para la innovación y la mejora de la calidad. En definitiva, y de acuerdo con Gómez, Roses y Farías (2012), las redes sociales contribuyen a impulsar un aprendizaje constructivista y colaborativo.

La idea es mejorar el proceso de aprendizaje empleando la atracción del colectivo de estudiantes por las nuevas tecnologías, a la par que se consigue una mayor implicación de estos derivada de la asunción de un papel protagonista dentro de su propio proceso formativo y del de sus compañeros de aula. No podemos olvidar que estamos ante un colectivo habituado al uso de estas herramientas (Del Fresno, 2011), o de lo que se ha dado en llamar «nativos digitales» (Prensky, 2001).

Ya ha quedado demostrado en varios estudios que el empleo de redes sociales, como apoyo a la formación, mejora los resultados de aprendizaje de los estudiantes (Saquete y otros, 2011 y Del Moral y Villalustre, 2012). Pese a estas conclusiones, hemos constatado que el uso de las redes sociales como una herramienta didáctica está reservada a experimentos aislados, así lo entienden, entre otros Dapía y otros (2014) y Poza, Calduch, Albors, Cabrera, Teruel, Rebollo y Díez (2014).

El objetivo es aprovechar el potencial de las redes sociales para estimular al estudiante, así como para promover aprendizajes significativos y optimizar el esfuerzo de todos los participantes en el proceso (Poza y otros, 2014). No en vano es una realidad que el uso de estas herramientas favorece la incorporación de profesionales al mercado laboral con el consiguiente beneficio que esto tiene para su inserción en un mercado dominado por los medios digitales (Vivar, García, Vinader, Núñez y Martín, 2012). Ahora bien, en medio de estas evidencias a favor del empleo de las redes sociales en el ámbito educativo, no es menos cierto que su utilidad y buenos resultados suponen un reto para los docentes que son quienes deben encontrar la manera de innovar e incorporarlas en el proceso de enseñanza y aprendizaje (Ricardo y Chavarro, 2011).

Cada red tiene sus rasgos característicos que le atribuyen un perfil más o menos idóneo para el desarrollo de determinadas actividades o tareas. Es por ello que el empleo combinado de varias redes diferentes permite optimizar los resultados globales, en términos de adquisición de competencias. De entre las muchas redes sociales existentes, en este proyecto optamos por el empleo de Facebook y Twitter, complementadas con Pinterest, si bien en este artículo nos centraremos en el uso de la primera de ellas. 


\subsection{La red social Facebook en el contexto universitario}

Facebook se posiciona como la plataforma digital más grande del mundo. Creada el 4 de febrero de 2004, cuenta con 1555 millones de usuarios activos por todo el planeta, llegando a convertirse en la preferida por los internautas, por delante de otras como Youtube, Twitter o LinkedIn. Cada usuario permanece una media de 20 minutos al día en la red, siendo la manera de acceso preferida por los mismos el dispositivo móvil, desde el que se conectan el 90\% de los usuarios totales.

En el colectivo formado por los estudiantes universitarios estas cifras subrayan las anteriores dado que Facebook es la red con mayor notoriedad y nivel de uso en dicho grupo; esta es precisamente una de las razones por las que hemos optado por su empleo en la docencia. Por otro lado, la elección de esta herramienta descansa en sus propias características y utilidades que la convierten en un recurso interesante para compartir recursos y comunicar información sobre una asignatura y los eventos vinculados a esta, en medio de un entorno que permite mantener una conexión y relación cercana y dinámica con el estudiante (Vivar y otros, 2012 y Alonso, Moreno, Terrones, Agulló y Juan, 2013).

La hipótesis general de la que partimos es que el uso de la red social Facebook logra mejorar el proceso de enseñanza y aprendizaje entre los estudiantes universitarios, además de facilitar la adquisición de ciertas competencias clave para el Grado en Administración y Dirección de Empresas y ampliables a otros estudios, entre las que destacan la capacidad de síntesis, manejo de las TIC y trabajo en equipo. Estudios previos concluyen resultados positivos derivados del uso de las redes sociales a las que atribuyen un gran potencial educativo (Espuny, González, Lleixá y Gisbert, 2011; Gómez y otros, 2012 e Iglesias y González, 2014, entre otros).

En el ámbito docente, Facebook proporciona diferentes utilidades para tratar de aprovechar sinergias en el trasvase de conocimiento desde el docente hacia sus estudiantes, así como al pretender aumentar la cohesión entre los mismos, creando y diseñando acciones formativas que en muchas ocasiones llegan más allá del aula. Por otro lado, en línea con investigaciones precedentes como Ellison, Steinfield y Lampe (2007), Abuín (2009) e Iglesias y González (2014), estamos convencidos de que el empleo de esta herramienta favorecerá la relación entre los estudiantes del grupo y entre estos y el profesorado, culminando en un escenario favorable al desarrollo del trabajo en equipo, o más bien colaborativo por las posibilidades que ofrece para compartir y aportar de forma constructiva. Otra de las premisas de las que partimos es el potencial que Facebook posee para hacer más atractivo el proceso de aprendizaje, por su carácter novedoso y por la capacidad creativa que aporta al estudiante.

Un ejemplo claro de utilidad de esta plataforma en el ámbito educativo es la herramienta «Grupos», con la cual podemos crear un lugar de reunión para nuestros estudiantes en el que compartir conocimiento y desarrollar acciones que permitan la obtención de las competencias necesarias para el aprendizaje de una determinada área de conocimiento.

$\mathrm{Al}$ igual que la anterior herramienta, Facebook pone a nuestra disposición la posibilidad de crear eventos, lanzar publicaciones informativas y preguntas relacionadas con el contenido o materia de estudio, fomentando la participación, animando al grupo y facilitando el proceso de aprendizaje de una manera activa y constante.

Otra acción que la plataforma pone a disposición del docente es la posibilidad de compartir enlaces a otros sitios en Internet, relacionados con los contenidos de la materia a impartir, pudiendo compartir con el estudiante publicaciones de profesionales provenientes de su blog o newsletter, enlaces a portales de video como Youtube o también de contenidos como Wikipedia entre otros. 
Por último debemos hacer mención a la facilidad que supone, tanto para el profesor como para los estudiantes, la posibilidad de manejar archivos y documentos relacionados con el aprendizaje de un determinado grupo, como pueden ser presentaciones de Power Point, infografías, mapas o cualquier otro archivo que resulte de utilidad para la comprensión de los contenidos por parte del alumnado.

\section{MATERIALES Y MÉTODOS}

\subsection{Diseño}

Antes de iniciar la actividad dentro de la red social Facebook valoramos las diferentes alternativas que ofrece la plataforma al objeto de seleccionar aquella que mejor se adaptase a los objetivos del trabajo. Entre crear una página de acceso libre y un grupo cerrado optamos por esta última alternativa. Ya en una experiencia anterior habíamos trabajado con Facebook y encontramos la reticencia de ciertos estudiantes a participar en un foro abierto al que podía asomarse cualquier colectivo. Además, la propia definición de grupo responde a los objetivos planteados dado que son un espacio pensado para que las personas intercambien opiniones acerca de intereses comunes. Ello nos llevó a proponer, para la segunda edición, un grupo cerrado en el que solo participasen los docentes de la asignatura, profesionales o invitados a ella por diferentes cuestiones, y los estudiantes matriculados.

El papel del docente fue en todo momento de dinamizador de la plataforma, además de «guardián» de las publicaciones, así como de las dudas y cuestiones que se pudieran plantear. En definitiva podríamos resumir su papel como «moderador activo», puesto que además de regular y ordenar la participación de los estudiantes, asumió el papel de potenciador y creador de debates de interés. Ahora bien, la plataforma permite asignar de forma continuada o temporal, el papel de moderador a aquellos miembros que se considere oportuno para contribuir a la activación del grupo.

La asignatura elegida para la realización del experimento fue Dirección Comercial II, obligatoria de tercer curso del Grado en Administración y Dirección de Empresas, pero puede ser aplicable a cualquier otra de este u otros Grados. La elección responde tanto al contenido y competencias que se trabajan en dicha asignatura, como a la madurez del estudiante, referida al nivel de conocimiento de los contenidos básicos de la materia y a su manejo de herramientas didácticas. Un estudiante de tercer curso ha adquirido un importante número de competencias y destrezas que le capacitan para sacar un provecho adecuado de herramientas como las redes sociales.

Antes de proceder al desarrollo de las actividades asociadas a la asignatura mencionada se dividió la clase, formada por 45 estudiantes, en grupos de 5 personas. A partir de ese momento, cada grupo distribuyó los roles entre sus miembros, así como el trabajo a realizar siendo la primera tarea la elección de un producto sobre el que realizar su plan de marketing.

Se pretendió en todo momento que el estudiante asumiese el rol de profesional que trabaja en equipo, de este modo se procuró una mayor implicación de cada grupo en su proyecto de producto. Así las cosas, el proyecto se estructuró en tres etapas. En la primera se diseñó el grupo de Facebook y se invitó a participar a todos los estudiantes matriculados y a dos profesionales externos. En la segunda se desarrollaron diferentes actividades como eventos, debates, foros de dudas, etc. que se nutrieron de las aportaciones de todos los integrantes del grupo. En la tercera pusimos en marcha el análisis de resultados. 


\subsection{Objetivos}

Los objetivos que nos planteamos con este trabajo se pueden sintetizar en los siguientes:

- Analizar la utilidad de las redes sociales, Facebook, en el ámbito educativo universitario, sobre todo como herramienta incentivadora y facilitadora del intercambio de conocimiento entre iguales.

- Formar a los estudiantes en el uso de las redes fuera del ámbito lúdico y así mostrar su potencial como herramientas de corte profesional.

- Conformar una red de recursos online que ayude a generar conocimiento sobre plan de marketing que pueda servir de ayuda a futuros estudiantes y profesionales.

- Mostrar la utilidad del trabajo colaborativo a través de las TIC, tanto para la mejora continua como para el logro de resultados óptimos.

\subsection{Uso y actividades vinculadas a la herramienta Facebook}

El uso de Facebook dentro de la asignatura Dirección Comercial II fue complementario al resto de actividades y herramientas empleadas en el proceso de enseñanza-aprendizaje. Se trataba de combinar de una forma natural y nunca forzada, los ámbitos online y offline.

El carácter actual y práctico de la asignatura favorecía la integración de diversas actividades profesionales dentro de la actividad docente habitual. Nos referimos básicamente a: conferencias relativas a temas de interés y actualidad, visitas de profesionales al aula para compartir procesos, formas de actuar y conocimiento de empresa y excursiones fuera de la Facultad que permitieran conocer in situ el día a día de una empresa en el ámbito comercial. Para optimizar el aprendizaje e implicación del estudiante en relación con estas actividades, el uso de la red social Facebook se reveló como un posible aliado que permitía trabajar antes de su desarrollo, durante el mismo y con posterioridad. Así, antes del desarrollo de cada actividad se empleó la red social para proponer cuestiones de debate, para tratar de despertar mayor interés entre los estudiantes que trataban de aportar noticias o cuestiones actuales relativas al tema a tratar o a la empresa a visitar. Ello permitía que los estudiantes acudiesen al evento informados, lo que les permitía plantear cuestiones a los profesionales que enriquecieran los encuentros. Nuestro propósito fue que todo ello abocase en una mayor implicación de los estudiantes con cada actividad. El feed back posterior pretendía hacer balance de la utilidad e interés de la jornada.

Por otro lado, en el día a día de las clases presenciales estaba previsto un elevado protagonismo de los estudiantes que tendrían que compartir con sus compañeros los avances realizados en cada momento. Facebook permitía compartir antes de cada clase el trabajo, de modo que el resto de compañeros pudieran analizarlo y hacer aportaciones y sugerencias que luego fuesen debatidas en el aula. De este modo se lograba una perfecta interacción entre entorno online y offline que también tuvo su fiel reflejo en la constante publicación de contenidos de interés por parte de docente y estudiantes, al hilo de las cuestiones teóricas que se iban analizando en cada una de las clases. Todo ello permitió que los estudiantes se implicasen, no solo con su grupo de trabajo y en su rol de aprendizaje, sino también con el resto de proyectos y en un rol más activo, capaz de contribuir al desarrollo intelectual del equipo.

La Tabla 1 recoge de forma sintética las diferentes actividades propuestas, las acciones desarrolladas en Facebook, en consonancia con aquellas, y los beneficios perseguidos para el estudiante. 


\begin{tabular}{|c|c|c|c|}
\hline & ACTIVIDAD & ACCIÓN DESARROLLADA & BENEFICIOS PERSEGUIDOS \\
\hline \multirow{4}{*}{$\begin{array}{l}\text { Actividades comple- } \\
\text { mentarias, dentro y } \\
\text { fuera del aula }\end{array}$} & Ciclo de conferencias & \multicolumn{2}{|l|}{ Acción previa: dinamización } \\
\hline & \multirow{2}{*}{ Jornadas de la empresa en el aula } & $\begin{array}{l}\text { Planteamiento de cuestiones a re- } \\
\text { solver }\end{array}$ & $\begin{array}{l}\text { Incentivar } \\
\text { Implicar }\end{array}$ \\
\hline & & $\begin{array}{l}\text { Aportar información sobre tema } \\
\text { y/o empresa }\end{array}$ & \multirow{2}{*}{$\begin{array}{l}\text { Despertar interés, motivación } \\
\text { Asumir roles }\end{array}$} \\
\hline & Visitas a empresas & Creación de eventos & \\
\hline \multirow{6}{*}{$\begin{array}{l}\text { Exposición de trabajos } \\
\text { en clase, trabajo pre- } \\
\text { sencial }\end{array}$} & \multirow{2}{*}{$\begin{array}{l}\text { Borradores sobre cada parte del plan } \\
\text { de marketing (entorno, producto, pre- } \\
\text { cio, distribución y comunicación }\end{array}$} & \multicolumn{2}{|l|}{ Plantear dudas } \\
\hline & & $\begin{array}{l}\text { Proponer acciones de mejora para } \\
\text { otros grupos }\end{array}$ & Ayuda y colaboración \\
\hline & \multirow{4}{*}{$\begin{array}{l}\text { Informe definitivo sobre cada parte } \\
\text { del plan de marketing }\end{array}$} & & Alentar de igual a igual \\
\hline & & $\begin{array}{l}\text { Plantear posibles lormas de coo- } \\
\text { peración }\end{array}$ & Aprender de otros \\
\hline & & $\begin{array}{l}\text { Aportar documentos online ad } \\
\text { hoc para ayudar a otros grupos }\end{array}$ & $\begin{array}{l}\text { Desarrollo de trabajo colabora- } \\
\text { tivo }\end{array}$ \\
\hline & & Crear eventos & \\
\hline \multirow{5}{*}{$\begin{array}{l}\text { Actualidad y trabajo } \\
\text { diario }\end{array}$} & \multirow{5}{*}{$\begin{array}{l}\text { Noticias relacionadas y asuntos de in- } \\
\text { terés }\end{array}$} & \multirow{3}{*}{$\begin{array}{l}\text { Compartir recursos de interés } \\
\text { para la parte objeto de estudio en } \\
\text { cada momento: vídeo, imágenes, } \\
\text { documentos, etc }\end{array}$} & Ayudar de igual a igual \\
\hline & & & Compromiso con otros \\
\hline & & & Implicación y aliento \\
\hline & & $\begin{array}{l}\text { Compartir herramientas para el } \\
\text { desarrollo de los trabajos }\end{array}$ & Gestores de contenidos \\
\hline & & Compartir eventos & \\
\hline
\end{tabular}

\section{ANÁLISIS Y RESULTADOS}

La medición de resultados se hizo de tres formas diferentes. Por un lado se empleó la herramienta Grytics que nos permitió conocer diferentes parámetros de participación cuantitativa de los miembros del grupo a lo largo del periodo de desarrollo del experimento. De otra parte, realizamos un análisis de contenido de las aportaciones realizadas por los estudiantes, al objeto de complementar los datos numéricos aportados por Grytics. Finalmente empleamos la dinámica de grupos en combinación con la entrevista en profundidad a los participantes, para tratar de conocer su opinión de la red social, así como su utilidad percibida como herramienta de apoyo en el proceso de aprendizaje. 


\subsection{Análisis numérico a través de los parámetros de Grytics}

A través de la citada herramienta se han recogido datos históricos de cada una de las semanas en las que se aplicó el experimento: las quince semanas lectivas de duración del cuatrimestre más las dos subsiguientes en las que se finalizaron los trabajos definitivos. Los parámetros empleados fueron: reacciones, posts, comentarios, miembros activos, engagement score y activity score. Las reacciones son un indicador más endeble que simplemente recoge «me gusta» o similares. Con el post medimos el número de publicaciones que realiza el grupo en el periodo considerado, mientras que los comentarios son respuestas o aportaciones a un post determinado; se trata de dos elementos de gran valor para el posterior análisis de contenido. Otro elemento considerado es el número de individuos dentro del grupo que han permanecido activos dentro del intervalo analizado. El indicador engagement score mide el nivel de compromiso e implicación de los miembros del grupo. Finalmente, el indicador activity score se determina del siguiente modo (Figura 1).

\section{Figura 1}

\section{Estadísticas básicas de Gritycs para el grupo MarketingCET}

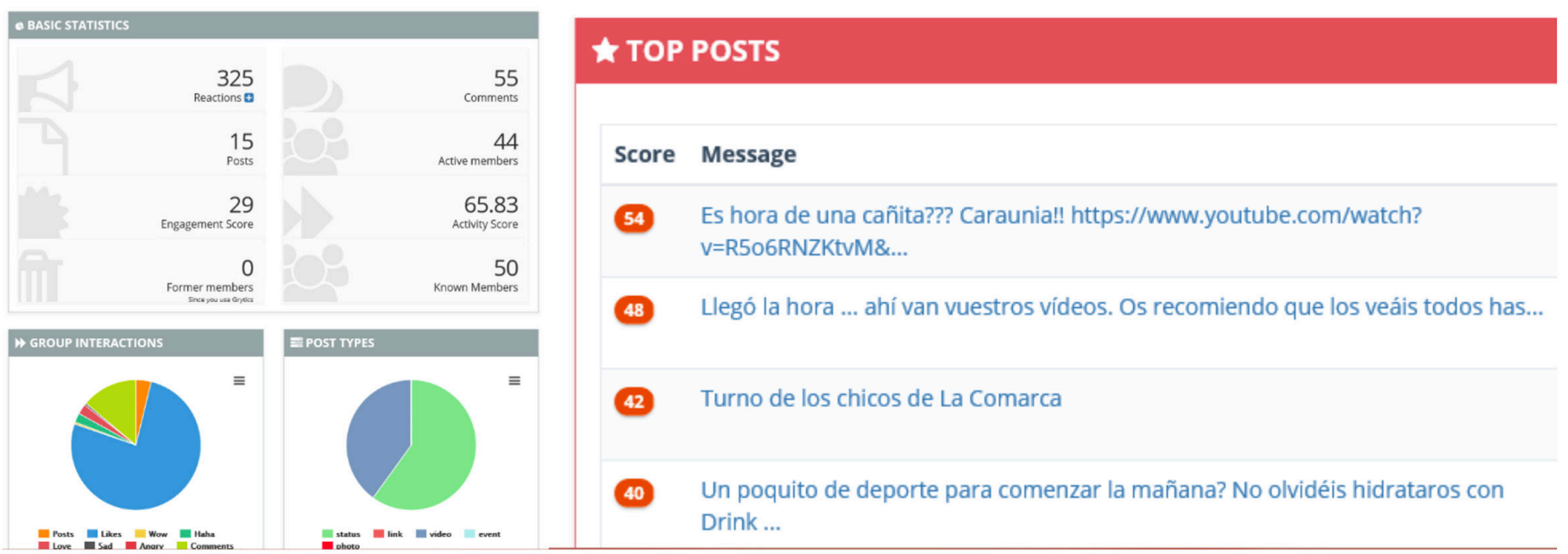

\subsection{Análisis de contenido}

Dividimos el análisis de contenido en los siguientes ítems: post, reacciones, eventos, asistencia y dinamización y comentarios. La Tabla 2 recoge cada ítem con su descripción. 
Tabla 2

Ítems del análisis de contenido.

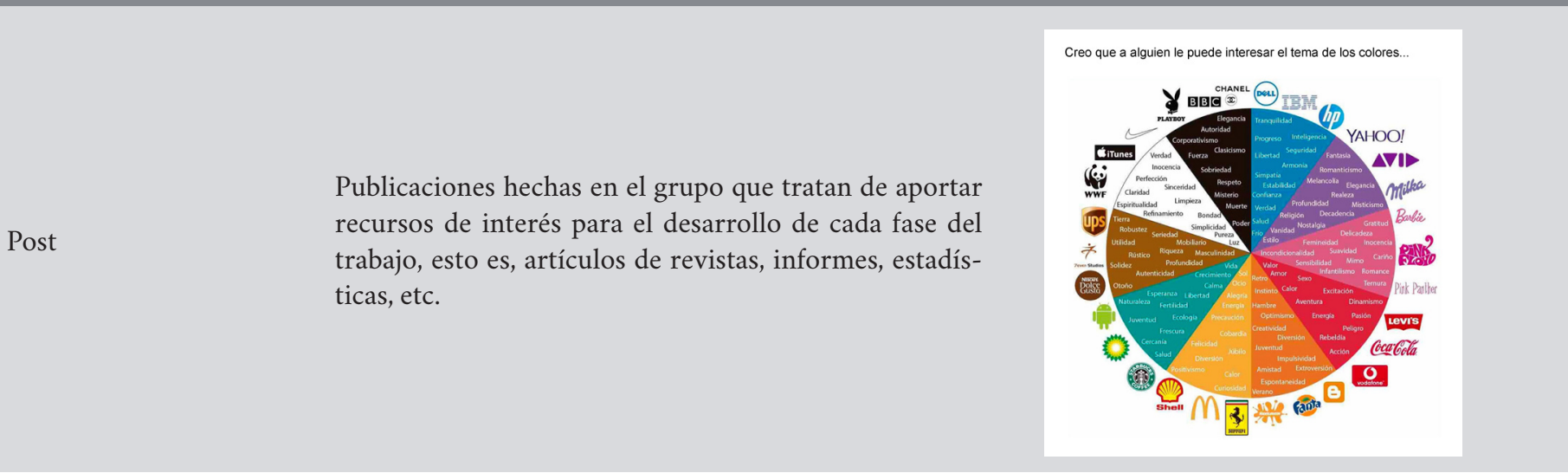

0012

B 3 Me gusta
2 comentarios Visto por 77

Comentar

Terminamos un fin de semana muy fructífero para nuestro sector. Tras una gran https://m. facebook.com/Feria-de-la-Trufa-de-Abejar-1631012.../ con infinidad de ponencias, charlas y demostraciones. Volvemos a casa con amplios conocimientos y muchas ideas nuevas para aplicar a nuestros productos

Asistencia ydinamización

Publicaciones que plantean dudas puntuales o que comentan cuestiones de la clase presencial de ese momento, por ejemplo en forma de resumen o conclusión de lo explicado

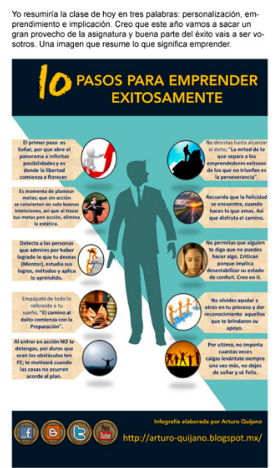

Comentarios
Se ha creado un evento como jornada, visita a empresas o conferencia, entre otros
Teresa Martínez Galán Muchas gracias Vicky Orden Avilés, tendremos en cuenta el artículo! Te mando un enlace que acabo de ver sobre packaging y su importancia, en este caso el producto son muy originales.

http://www. puromarketing.con/.../eggs-packaging-tiendes

Aglutina las reacciones en forma de aportación a los post de los miembros del grupo
David Palacin Arce Me parece muy interesante y como consumidor estoy totalmente de acuerdo, os dejo un reportaje de EL MUNDO que leí hace ya un tiempo y que demuestra que las empresas que que lei hace ya un tiempo y que demuestra que las empres
destacan ponen en valor el servicio de atención al cliente: 


\subsection{Análisis cualitativo. Entrevistas en profundidad y dinámica de grupos}

Se realizaron dos reuniones de grupo y tres entrevistas en profundidad. El objetivo fue extraer información sobre la utilidad de la red para su proceso de aprendizaje, así como para el desarrollo de ciertas competencias del Grado. Los temas tratados estuvieron relacionados con el manejo de la herramienta y su aplicabilidad en el aula, con su utilidad para fomentar el empoderamiento y participación de los estudiantes, su interés como instrumento de apoyo en el trabajo colaborativo y su uso como herramienta de resolución de dudas tanto teóricas como relativas al proceso de diseño de su trabajo.

\section{DISCUSIÓN Y CONCLUSIONES}

En general observamos que el uso de una red social en el desarrollo del proceso de enseñanza-aprendizaje es positivo para los estudiantes, quienes están habituados a relacionarse con sus iguales de este modo. En este sentido nos dicen que para ellos es cómodo y supone operar en un entorno «amigable». Además, el saber que sus comentarios no son públicos, sino que quedan dentro del entorno del aula les permite expresar dudas y contenidos relativos a la asignatura de una forma más adecuada.

La red social sirve además para compartir materiales, prácticas y recursos que favorezcan el aprendizaje. En general los alumnos coinciden al señalar el beneficio derivado del uso de Facebook en el proceso de aprendizaje.

Por otro lado, constatamos que el grupo creado ha sido útil para implementar el uso colaborativo de la red social, además de mejorar el proceso de enseñanza-aprendizaje. En buena parte esto se debe a que la productividad del estudiante se incrementa cuando trabaja en un grupo que trata de conseguir el mismo objetivo. Por otro lado y no menos importante, el trabajo en grupo permite complementar las carencias de unos con los puntos fuertes de otros y así avanzar en diferentes áreas.

El uso de la red como instrumento para plantear dudas, tanto al propio profesor como al resto de los compañeros, han sido factores determinantes para que el alumnado perciba como útil la creación de este grupo: mayoritariamente admiten que ha mejorado las relaciones entre sus iguales, así como con los profesores ya que esta herramienta les hace más accesibles y cercanos.

Como afirma Duart (2009), la verdadera transformación se encuentra en la dinámica educativa, en el proceso educativo que se desarrolla en el aula y, hoy cada vez más, fuera de ella. El uso activo y social de las redes no puede ser ignorado en las planificaciones docentes, aunque debe reconocerse que requiere de una mayor dedicación por parte del profesorado fuera del aula.

Lo que está claro es que las posibilidades para la formación y enseñanza que Facebook nos presenta en la actualidad son muchas y, con inventiva e innovación educativa, dichas posibilidades pueden ser muchas más, haciendo mucho más completo el proceso de aprendizaje y aumentando las competencias adquiridas por el estudiante en dicho proceso. 


\section{Apoyos}

Se enmarca dentro de la convocatoria 2016/17 de Proyectos de Innovación Docente del Vicerrectorado de ordenación académica de la Universidad de Valladolid.

\section{REFERENCIAS}

Abuín, N. (2009). Las redes sociales como herramienta educativa en el ámbito universitario. Revista Electrónica de ADA-Madrid, 3,199-205.

Alonso, M., Moreno, J., Terrones, A., Agulló, J. y Juan, A. (2013). Una experiencia docente universitaria a través de la red social Facebook. En XI Jornadas de Redes de Investigación en Docencia Universitaria, (pp. 331-341). Universidad de Alicante.

Amador, P. y Amador, J. (2014). Academic advising via Facebook: Examining student help seek-ing. Internet and Higher Education, 21, 9-16, doi: https://doi.org/10.1016/j.iheduc.2013.10.003

Balula, A. y Moreira, A. (2014). Evaluation of online higher education learning, interaction and technology. Suiza: Springer.

Cobo, C. y Pardo, H. (2007). Planeta Web 2.0 Inteligencia colectiva o medios fast food. Barcelona: Grup de Recerca d’interaccions Digitals. Universitat de Vic y Flasco México.

Dapía, M. y Escudero, R. (2014). Aprendizaje colaborativo mediante el uso de edublog en la enseñanza universitaria. Valoración de una experiencia. Enseñanza es Teaching, 32(2), 53-72.

De Haro, J. J. (2010). Redes sociales en la educación. Madrid: Anaya.

Del Fresno, M. (2011). Netnografía. Investigación, análisis e intervención social online. Barcelona: Editorial UOC.

Del Moral, M. E. y Villalustre, L. (2012). Didáctica universitaria en la era 2.0: competencias docentes en campus virtuales. Revista de Universidad y Sociedad del Conocimiento (RUSC), 9(1), 36-50. Recuperado de https://goo. gl/8Ydbu 8

Duart, J. M. (2009). Internet, redes sociales y educación. RUSC. Revista de Universidad y Sociedad del Conocimiento. 6(1), 1-2.

Ellison, N. B., Steinfield, C. y Lampe, C. (2007). The benefit of Facebook "friends:" Social capital and college students' use of online social network sites. Journal of Computermediated Communication, 12(4), 1143-1168, doi: https://doi.org/10.1111/j.1083-6101.2007.00367.x

Espuny, C., González, J., Lleixá, M. y Gisbert, M. (2011). Actitudes y expectativas del uso educativo de las redes sociales en los alumnos universitarios. Revista de Universidad y sociedad del Conocimiento, 1, 171-185.

Esteve, F. (2009). Bolonia y las TIC: De la docencia 1.0 al aprendizaje 2.0. La Cuestión Universitaria, 5, 58-67. Recuperado de http://polired.upm.es/index.php/lacuestionuniversitaria/article/viewFile/3337/3402 
Garrigós, I., Mazón, J. N., Saquete, E. Puchol, M. y Moreda, P. (2010). La influencia de las redes sociales en el aprendizaje colaborativo. En XVI Jornadas de Enseñanza Universitaria de la Informática. Universidad de Santiago de Compostela.

Gómez, M., Roses, S. y Farías, P. (2012). El uso académico de las redes sociales en universitarios. Revista Comunicar, 38 (XIX), 131-138, doi: https://doi.org/10.3916/C38-2012-03-04

Iglesias, M. y González, C. (2014). Facebook como herramienta educativa. Historia y Comunicación Social,1(19), 379-391. Recuperado de https://goo.gl/XNRsB8

Junco, R. (2011). The need for student social media policies. Educause Review, 1(46), 60-61. Recuperado de https:// goo.gl/5qgonH

León de Mora, C. (Coord.) (2010). Entornos colaborativos en docencia virtual: redes sociales y wikis. Sevilla: Secretariado de Publicaciones de la Universidad de Sevilla.

Lozano, A. (2004). Comunidades de aprendizaje en red: diseño de un proyecto de entorno colaborativo. Revista electrónica Teoría de la Educación: Educación y cultura en la sociedad de la información, 5. Recuperado de https:// goo.gl/ercxDV

Ortego, M. T., Jiménez, E. y Fernández, P. (2015). Linguistic behaviour in social networking sites used as academic and educative communication tool. Procedia. Social and Behavioral Sciences, 212, 123-130. Recuperado de https://goo.gl/1aErhP

Ponce, I. (2012). Monográfico Redes Sociales. Observatorio tecnológico. Ministerio de Educación, Cultura y Deporte. Recuperado de https://goo.gl/rd7Q28

Poza, J. L., Calduch, A., Albors, A., Cabrera, M., Teruel, D., Rebollo, M. y Díez, R. (2014). Propuesta de parámetros y caracterización de los grupos de las redes sociales orientados a la docencia universitaria: experiencia y resultados. Revista de Educación a Distancia (RED), 44, 2-18.

Prensky, M. (2001). Digital Natives, Digital Immigrants. On the Horizon, 9(5), 1-6. Recuperado de https://goo.gl/ ahLGiK

Ricardo, C. y Chavarro, A. (2010). El uso de Facebook y Twitter en educación. Revista Lumen, 11. Recuperado de https://goo.gl/qysvXG

Saquete, E., Garrigós, J., Mazón, S. e Izquierdo, R. (2011). Influencia de los estilos de aprendizaje en el uso de redes sociales para docencia. Recuperado de https://goo.gl/j1uTNu

Tomás, D. y Navarro, B. (2011). Las redes sociales como instrumento dinamizador del aprendizaje colaborativo. Recuperado de http://web.ua.es/es/ice/jornadas-redes-2011/documentos/posters/183087.pdf.

Valenzuela, R. (2013). Las redes sociales y su aplicación a la educación. Revista Digital Universitaria, 4(14). Recuperado de https://goo.gl/DQzXnT

Vivar, H., García, A., Vinader, R., Núñez, P. y Martín, M. A. (2012). La innovación educativa en la enseñanza superior: Facebook como herramienta docente. Revista de Comunicación Vivat Academia, febrero, 530-544. Recuperado de https://goo.gl/cVyPcZ 


\section{INFORMACIÓN SOBRE LAS AUTORAS}

Piedad Vargas Soria. Doctora en Historia del Arte (cum lauden) y Licenciada en Bellas Artes. Durante los últimos 14 años ha compaginado su actividad docente en la Universidad y Escuelas de Arte con la participación en ponencias, conferencias, congresos y grupos de trabajo. Todo ello, mientras continúa con su carrera artística (www. payevargas.com), además de organizar y coordinar eventos culturales y comisariar exposiciones. Su espíritu creativo e inquietud le ha llevado a una constante formación multidisciplinar y a la experimentación e investigación en el campo de la Historia del Arte, los procesos creativos y el Arte en general, realizando diversas publicaciones.

$\triangle$ mariapiedad.vargas@uva.es

Blanca García Gómez. Profesora Titular de Universidad en Universidad de Valladolid y Decana de la Facultad de Ciencias Empresariales y del Trabajo de la Universidad de Valladolid. Consejera de Caja Rural de Soria. Directora de la Cátedra Conocimiento e Innovación Caja Rural de Soria de la Universidad de Valladolid. Profesional docente e investigadora en diferentes áreas de marketing con el objetivo de desarrollar su faceta investigadora en ámbitos como el marketing digital y la estrategia de marketing en el sector vitivinícola. Especialista en investigación de mercados, análisis ad hoc mediante encuesta y desarrollo de planes de viabilidad de empresas. Ha desarrollado una potente línea de investigación centrada en la fidelización de clientes.

\section{bgarcia@eade.uva.es}

Elena Jiménez García. Licenciada en Filología Hispánica y Doctora en Lingüística española. Desde 2002 profesora e investigadora del Departamento de Didáctica de la Lengua y la Literatura. Ha realizado diversos estudios dialectológicos sobre el léxico agrícola, aspectos de comunicación no verbal, el humor y la creatividad lingüística y literaria, errores lingüísticos en la prensa, y estudios sobre antropología cultural, análisis del discurso y Hermenéutica analógica. Ha realizado estancias docentes y de investigación en 13 universidades europeas y en la City University of New York. Ha sido investigadora en varios proyectos de investigación y proyectos de innovación docente de la Universidad de Valladolid y de la Junta de Castilla y León. 